\title{
VIDANN: A video annotation system
}

\author{
ARMAND DE CLERCQ, ANN BUYSSE, and HERBERT ROEYERS \\ Ghent University, Ghent, Belgium \\ WILLIAM ICKES \\ University of Texas, Arlington, Texas \\ and \\ KOEN PONNET and LESLEY VERHOFSTADT \\ Ghent University, Ghent, Belgium
}

\begin{abstract}
VIDANN is a computer program that allows participants to watch a video on a standard TV and to write their annotations (thought/feeling entries) on paper attached to a writing tablet. The system is designed as a Microsoft ActiveX module. It can be further adapted by the individual researcherthrough the use of a VBScript. All data, including the participant's handwriting, are stored in an XML database. An accompanying Wizard has been designed that enables researchers to generate VBScripts for standard configurations.
\end{abstract}

People are typically motivated to understand each other's psychological states. Recent theoretical and methodological innovations have led to the development of a reliable and well-validated research paradigm for the study of "everyday mind reading." This empathic accuracy paradigm enables researchers to determine how accurately perceivers can infer the thoughts and feelings of target individuals in videotaped conversation sequences (Ickes, Stinson, Bissonnette, \& Garcia, 1990).

The method can be broken down into several phases (Figure 1). In a first phase, 2 participants are videotaped by means of a concealed camera while they are having a spontaneous interaction. In Phase 2, which occurs immediately after, the participants look at their own copies of the videotape and stop the tape at each point during the interaction at which they remember having had a specific thought or feeling. Typically, the VCR is stopped with a remote start/pause control. At each of their self-determined tape stops, the participants are required to write down on a standard coding form the specific content of the thought or feeling that they had at that point. In Phase 3, the participants are instructed to view the videotape a second time; during this viewing, the experimenter stops the tape for them at each of those points at which their interaction partner had recorded a specific thought or feeling. The interactants' task in this phase is to infer and write down the content of each of their partners' thoughts and feelings. Finally, several independent coders rate the degree to which the content of the perceiver's inference matches the con-

The authors thank Vicenç Quera and a second, anonymous reviewer for their valuable comments on a previous draft of this paper. Correspondence concerning this article should be addressed to A. De Clercq, Department of Applied Mathematics and Computer Science, University of Ghent, Krijgslaan 281 S9, B9000 Ghent, Belgium (e-mail: armand. declercq@ rug.ac.be). tent of the target person's actual subjective experience, in order to assess the perceiver's empathic accuracy (see Ickes et al., 1990, for more details).

VIDANN - the system to be described in the present article-was designed to computerize each of the phases in the empathic accuracy paradigm, in order to improve the efficiency of collecting the various thought/feeling data and to help ensure the validity and reliability of the resulting empathic accuracy measures. In particular, the system minimizes (1) the inaccuracies related to the use of a manual remote control system to stop a videotape at specific time points and (2) the time-consuming activity of scanning or transcribing numerous written records. Achieving both of these goals is well served by a computercontrolled system.

Because research participants (including, in some studies, ones with impaired cognitive functioning) are expected to use the system themselves, the system has been designed so that it can be used without direct contact with the computer. The participants watch a video on a standard TV and write their annotations (thought/feeling entries) on a paper attached to a writing tablet. The participants are able to start/stop the video by touching the button on the writing tablet. The control computer is placed out of sight of the participant. The experimenter can see the participant's writing as it appears on the experimenter's computer screen and restart the video if necessary.

\section{VIDANN}

\section{System Description}

In Phase 1 (see Figure 1), images from the video camera are output directly to the computer (Figure 2). A standard video camera is used with an S-VHS output connected to a video editing card in the PC. The PC runs Microsoft Windows 98 and uses a hardware MJPEG codec for com- 


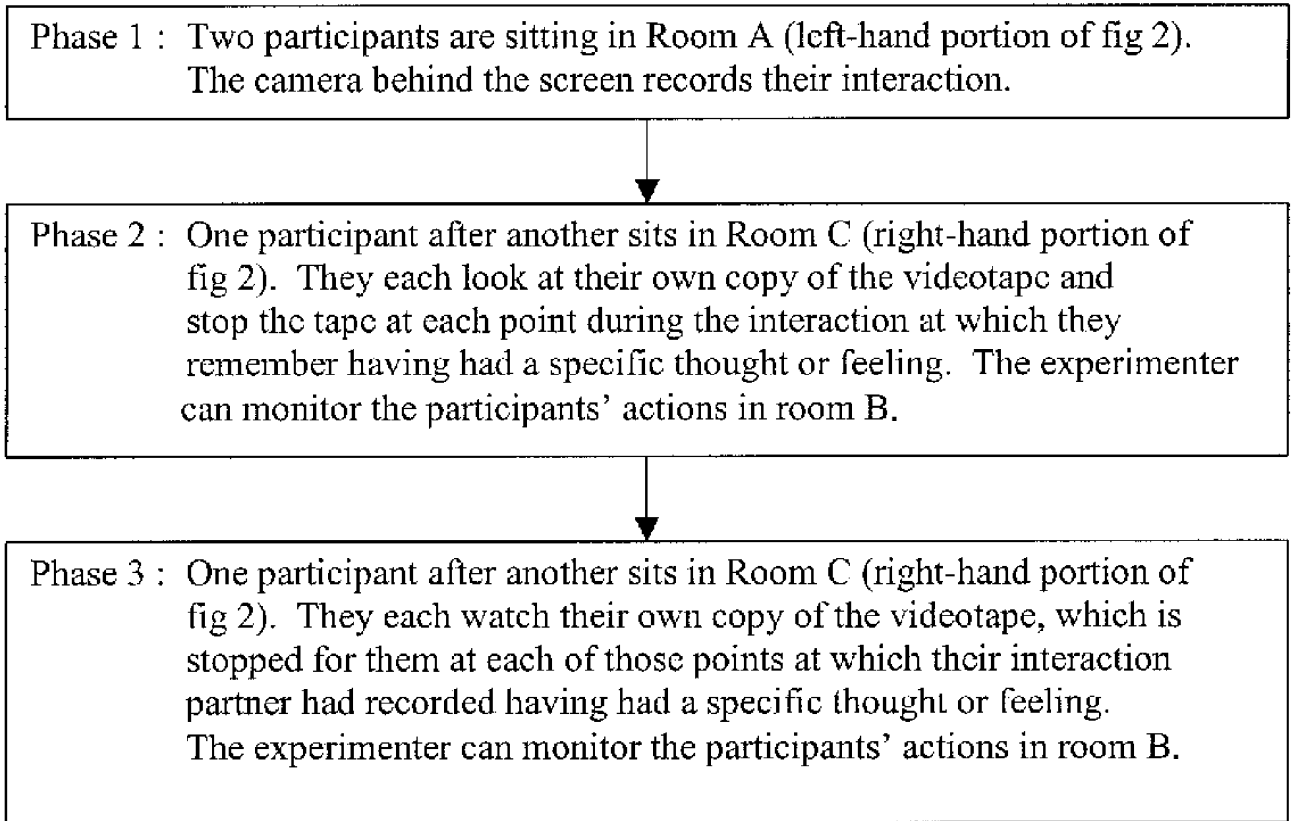

Figure 1. Scheme for the three phases.

pression of the images. We use a Pinnacle Systems MIRO DC10 Plus card for image compression, with a compression level of 10-15. With this level of compression, it is still possible to observe the interaction of the participants while maintaining a video data stream below $500 \mathrm{KByte} /$ sec. Furthermore, $30 \mathrm{~min}$ of video recording creates an
.AVI file of approximately $640 \mathrm{MB}$, which fits onto a single CD-ROM. Any video recording system can be used for this purpose as long as it can produce a video .AVI file that is "Video for Windows" compatible.

In the remaining phases of the procedure, the participant remains in the same room (Figure 2) while the video

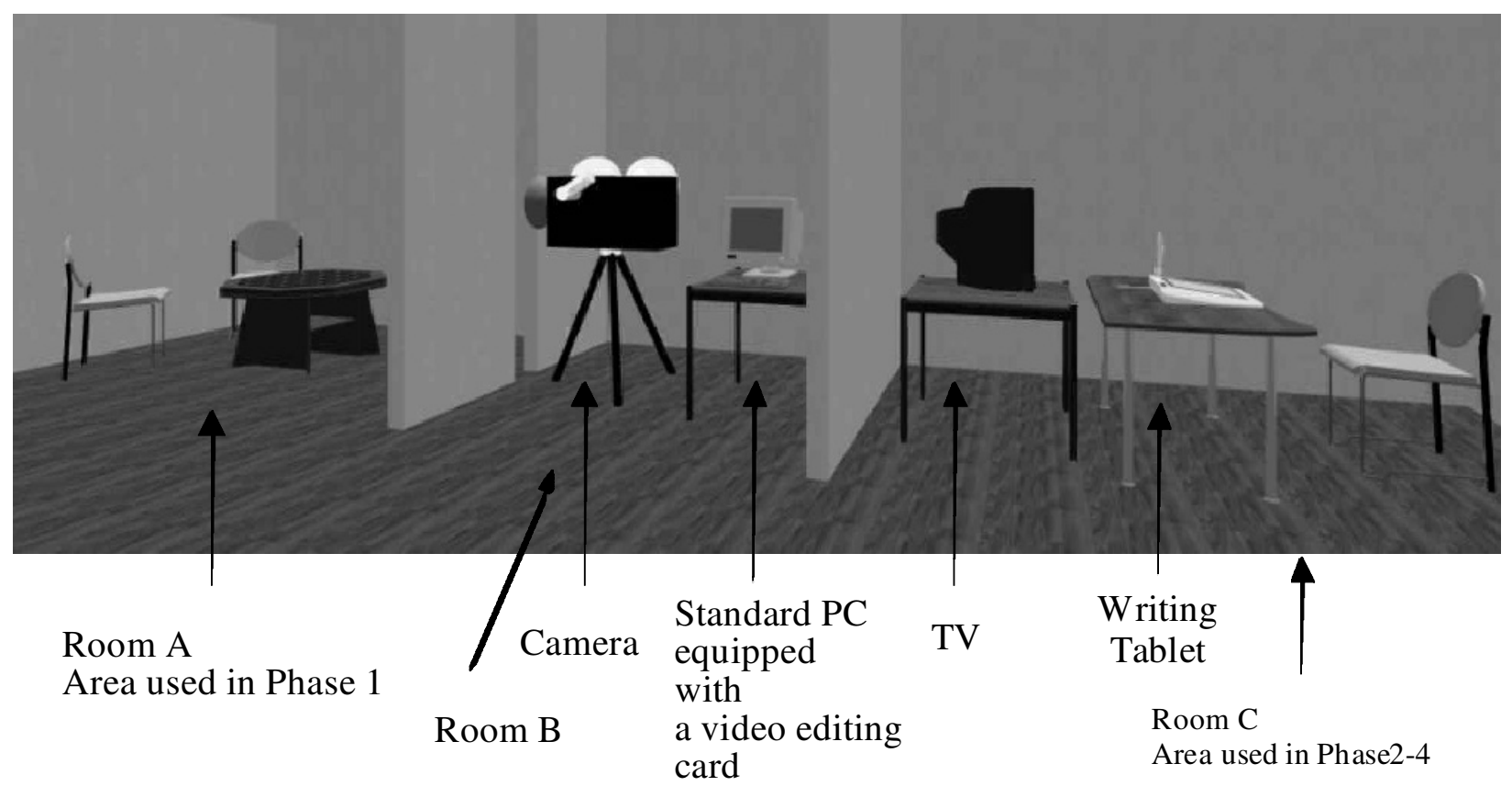

Figure 2. Equipment setup. 
is replayed. The same video editing card is used. Its composite VHS signal is connected to a standard TV set. The VIDANN software that is used in this part of the procedure is written in Visual Basic 6 (the relevant details will be explained later). The VIDANN software makes use of the Microsoft ActiveX Multimedia Control.

The PC that controls the hardware/software configuration for each participant is connected to the participant's writing tablet. The controlling software for the writing tablet is based on the LCS/Telegraphics WINTAB API driver that is configured for use in Visual Basic. WINTAB is a software standard, widely used for pointing devices. For this reason, a broad range of compatible tablets can be used without reprogramming the software.

The research participants can start and stop the video and still write their thoughts or feelings on the same kind of thought/feeling reporting form that was designed for use in the original empathic accuracy study (Ickes et al., 1990). The only real differences are that they write with a special ink pen and that the empathic inference form is attached to a Wacom writing tablet. There can be a maximum distance of $1 \mathrm{~cm}$ between the tablet surface and the paper on which the participant's thoughts and feelings are recorded. This makes it possible to attach a pad of paper to the tablet with pages that can be pulled off at the top as they are completed. (In fact, the actual paper form is not used anymore once the electronic version of the participant's handwriting has been stored. It is just there for the convenience of the user.)

The data from the participant's written responses are saved in a special format on the computer, as will be explained later. On the participant's writing tablet is a button (a reserved place, or location, that they can click with the pen). During Phase 2, a pen-click at this location acts as a start/stop button. During Phase 3, when the other partner has to write an inference about what the first partner was thinking and feeling, a pen-click on this button stops the tape precisely at each of the tape stops that were previously designated by the first partner. ${ }^{1}$ The controlling computer is placed out of the direct sight of the participants in order to minimize their awareness that a remote computer is being used.

Trained data coders can use the system in the same way that the research participants in Phases 2-4 do (Figure 3).
However, when trained data coders use the TV and the video card with the hardware compression codec, the preferred configuration is one in which a software MJPEG codec from Morgan Multimedia is used. In this configuration, the video is replayed in a window on the data coder's computer screen. This configuration has the advantage of not requiring any extra video hardware. It is clearly desirable, however, to use a fast Pentium II or a Pentium III to prevent missing frames during this type of replay presentation.

\section{Data Storage in XML Format}

All data, including those from the writing tablet, are stored in XML format, the extensible mark-up language. $\mathrm{XML}$ is a computer language designed to specify the structure of the data in a text file. It was developed under the auspices of the World-Wide Web Consortium (W3C; Cover, 1999). As in the well-known Web language HTML, XML employs the concept of generic mark-up. Tags are inserted into a document in order to specify its structure.

XML is intended to be a universal language for data exchange. It is already widely used in clinical applications (Okstein, 1999) and offers many exciting possibilities for psychological researchers (Stockburger, 1999). The following lines contain some text of an XML file as produced by VIDANN:

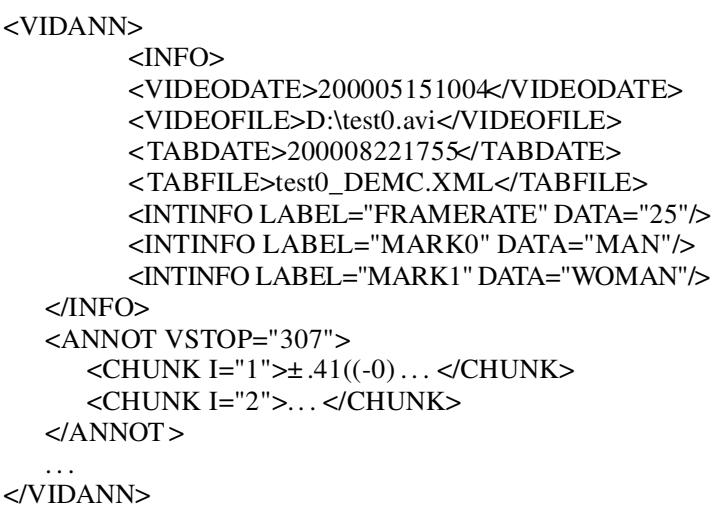

The part of the program that appears between the $<$ INFO $>$ and the $</$ INFO $>$ tags contains data concerning the experiment and the video file, such as creation date (between $<$ VIDEODATE $><$ /VIDEODATE $>$ ), file name,

The videotape of each participant is copied to a CD-ROM

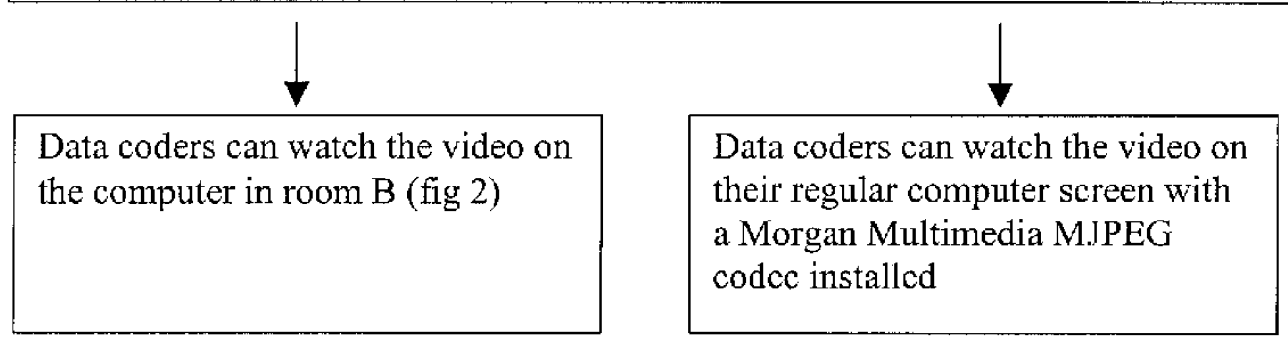

Figure 3. Scheme for the data coders. 
frame rate, and so on. The subsequent part of the program contains a number of $<$ ANNOT VSTOP ...</ANNOT $>$ blocks. Each block represents the data that belong to one particular video segment.

The VSTOP tag identifies the stop time. One or more CHUNK blocks follow. The code in each CHUNK contains all the pen movements that the participant has written on the writing tablet. A special binary format is used in which only the characters between 40 and 255 are valid. The first character in the text between $<$ CHUNK I="1"> and $</$ CHUNK $>$ : " $\pm .41((-0) "$ actually represents the byte code 241 . This means that the next 8 bytes represent $x$ and $y$ coordinates for the current pen position. If 40 is subtracted from every byte that follows, this results in 6,12 , $9,0,0,5,8$, and 1 . Every byte then represents 4 bits of the 16-bit coordinate value. This restriction in the character range ensures that there will be no interference with the special XML tag characters. As the example shows, the XML format makes it possible to view relevant data with an ASCII text editor for identification purposes without the need for a special XML editor. Storing the actual pen movements, instead of representing the data of one paper page in a bitmap, offers the data coders the possibility of resizing the window containing the written text and the pen thickness without any loss of resolution.

A final aim is to add time code to these data in the future. A time-code feature will make it possible for researchers to detect those places at which the participants stopped or hesitated before expressing thoughts and feelings in sentence form on the writing tablet.

\section{The Software Description}

VIDANN is written in Visual Basic 6. The Microsoft XML Parser Redistributable that comes with IE5 was used for programming VIDANN (Spencer, 1999). This means that the XML data are presented in the program as a series of objects, known as the DOM model. The XML data themselves are readable with either a normal text editor or a freeware XML editor, such as Microsoft XML notepad. The VIDANN system is created not as a standard .EXE file, but as an ActiveX.exe file.

VIDANN uses two objects that can be used in any other program under Windows. The first object, GO, is the principal object, and its methods perform the VIDANN tasks. The second object, MARK, generates the timing start/ stop codes. MARK contains an array with the stop frames from the video. These data are used to synchronize the video display and the client annotations on screen. Its main purpose, however, is to enable the user to generate stop signals "at random." For this reason, a second instance of the MARK object is generated that contains a randomized collection of the same stop frames. This second information is then used for the display of the client annotation. As such, the coder always sees a client annotation that never belongs to the actual video sequence displayed. This feature is used in tests of the data coders' reliability (Ickes et al., 1990).

The GO and MARK objects are used in a Script file written in VBScript (Visual Basic Script). This script can be freely modified according to the specific demands of a project, without the need to use an extra compiler or to reprogram VIDANN. The Windows Scripting Host from Windows 98 executes the script file. The system is thereby made sufficiently modular that experimenters can create standard annotations and codings for statistical analyses.

The user can choose between three different input modes: writing tablet, text typed directly into the computer, or categorical data selected by predefined parameters. For this last option, which is used by the coders, the number, the name, and the collection of possible answers for every parameter can be generated by the user with a separate program. For post hoc coding, the user can select up to four input screens at the same time for the display of handwriting and/or ASCII text. All windows can be placed and resized freely. The use of an executable script file makes it possible to select all necessary options before the experiment begins. For this reason, decision errors during the experiment will be kept to a minimum.

Figure 4 shows a particular screen for the Coder, executing the VBScript Coder.VBS. The code for this script is shown in the Appendix. In the first part of this script, constants and variables are defined, and the object GO is created. The necessary parameters for VIDANN, such as the name of the program directory, the definition of the different windows that will be used, the name of the video file, the startup message, and so forth, are defined by calling different subroutines (methods) from the GO object. The main part of the script consists of a loop in which GO methods are used for the playback of parts of the video and for reading the text from the writing tablet.

The concept of using VIDANN as a set of COM objects in a VBScript has two major advantages. First, it keeps the system transparent and modular. Each method of the GO object calls a set of classes and forms in VIDANN that are kept as independent of each other as possible. Second, it offers the experimenter the possibility of creating additional VBScript program lines without the need to reprogram VIDANN. The original Script file consists of nearly 40 program lines. Care has been taken to use long meaningful names for function and constant definitions. It is possible for an experimenter with a minimal knowledge of the VBScript language to adapt the original script to his or her particular needs. Additional messages to the user can also be added.

\section{The VIDANN Wizard}

Although the script looks quite simple, considerable programming knowledge is needed to adapt it to the purposes of a particular study. Because a typical psychological researcher cannot be expected to possess such knowledge, a Wizard has been created that enables the user to specify in the appropriate code all of the necessary parameters for his or her experimental conditions.

The idea of using a Wizard — or front-end editor-for generating code is not new. Most people do not write HTML code directly but, instead, use a generator, such as FrontPage or Dreamweaver. The same method is also used in E-Prime (Schneider, 1998), for which E-Studio 


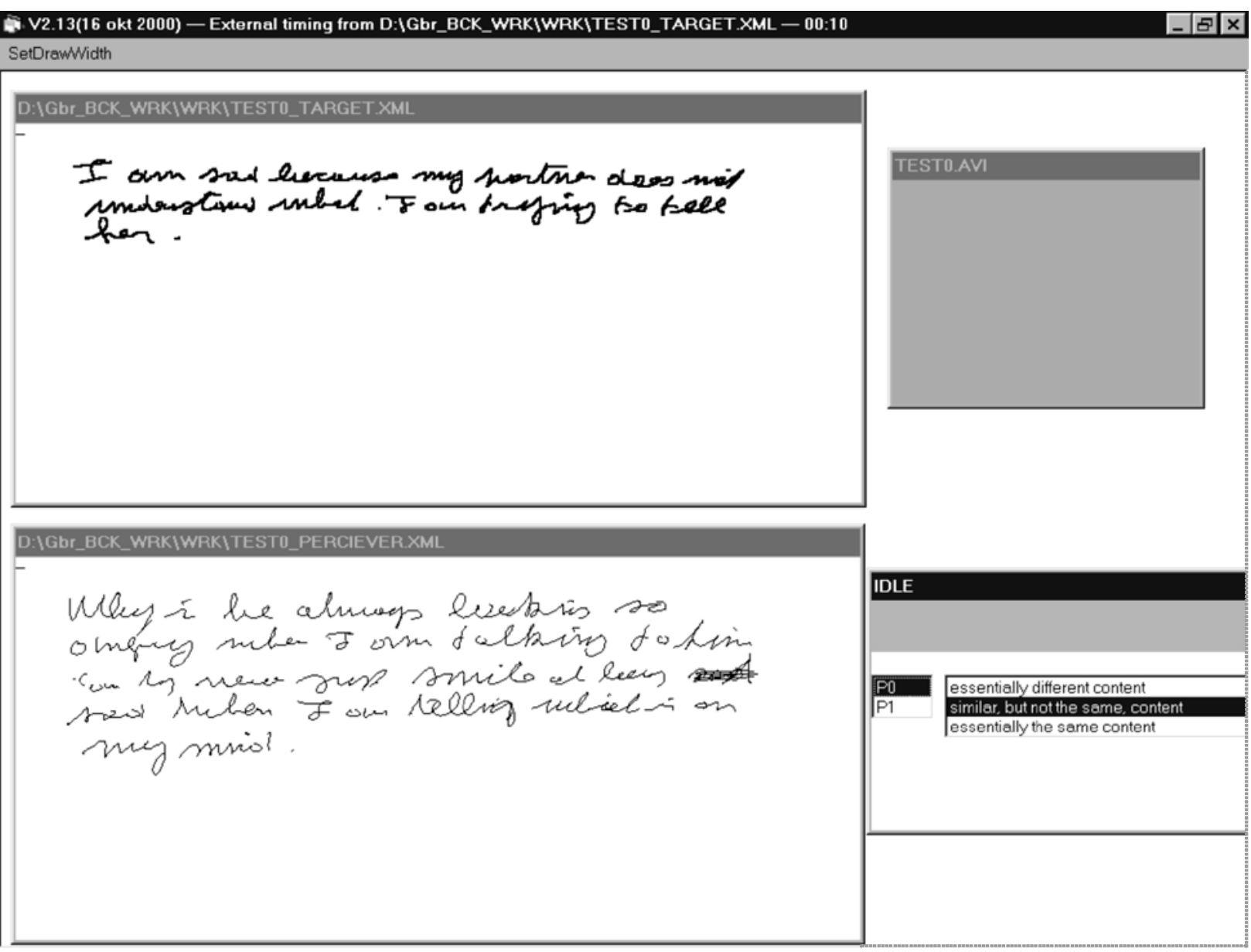

Figure 4. The VIDANN window for the data coder.

is a comprehensive, graphic, experimental design environment that generates code in E-Basic, a full-featured object-oriented Basic compiler with many enhanced commands for behavioral research. Essentially the same method was used for EPA2000 as well (De Clercq, Desoete, \& Roeyers, 2000).

The first step for generating an experiment's specified parameters with the Wizard is creating a script frame in XML. The appropriate XML files have already been created for those experiments for which VIDANN is being used at this moment. For other experiments, the user has to create a new XML file. The best way to do this is to make a copy of an existing XML file and then open it and make the appropriate revisions with the XML notepad editor. The following example is a part of the XML file that generates just one line in the VBScript. Figure 5 is a representation of the same XML segment in Microsoft XML notepad, a freeware XML editor. With the XML notepad editor, it is possible to modify the text, to add new blocks by copying and pasting existing blocks, or to delete blocks. The notepad editor will then generate the XML file structure as shown below.

$<$ LINES NR="4.2" $>$

$<$ TEXT $>$ GO.MessageFile $\$ 1</$ TEXT $>$ $\begin{array}{lr}\text { <ARG N="1"> } & 3 \\ \text { <INFO }>\text { This is the standard English message } & 4 \\ \text { file. Change the name if you have a file in } & 5 \\ \text { a different language }</ \text { INFO }> & 6 \\ \text { <CATEGORY }>\text { \$TXT(MessageEN.XML) }</ \text { CATEGORY }> & 7 \\ \text { ARG }> & 8 \\ \text { SES }> & 9\end{array}$ $</$ LINES $>$

The researcher must create one block for every line in the VBScript (see the Appendix for a VBScript example). The NR tag in Line 1 indicates the VBScript line number. The text between the $<$ TEXT $>$ and $</$ TEXT $>$ tags (Line 2) represents the script line in which parameters are represented with symbols such as $\$ 1, \$ 2 \ldots$ The next lines contain the description for each $\$$ argument. Each argument is described between the $<\mathrm{ARG}>$ and the $</ \mathrm{ARG}>$ tags. Between $<$ INFO $>$ and $</$ INFO $>$ appears the text that will pose the relevant queries to the user of the Wizard. \$TXT (Line 7) tells the Wizard that this is a text argument, with the default text appearing between the () brackets. Other arguments are also possible, such as \$KEY for selecting a start/stop key, \$NUM for a number (this is an argument without quotes), and \$Dir for selecting a file with a browser. We should emphasize, however, that researchers must understand the VBScript programming language if they wish to make such a script from scratch. 


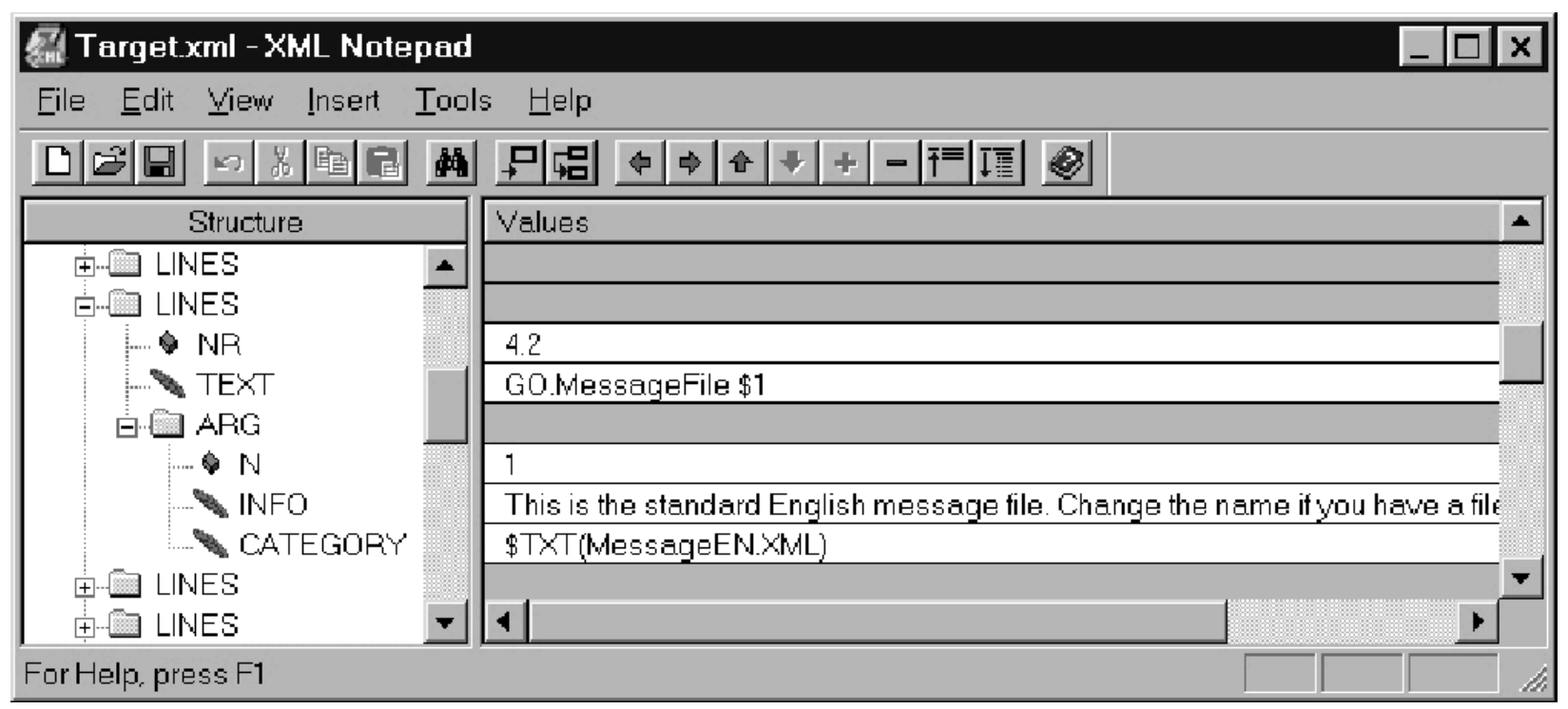

Figure 5. The XML notepad window.

In most cases, however, they will need only to change a few parameters or to modify/translate the text that will be displayed to the user of the Wizard (Lines 4-6 in the example above). In this case, the text can be easily changed by using the XML editor.

Each XML file also contains information denoting the kind of experimental condition for which it is intended. The Wizard starts by presenting a list of possible experimental conditions whose general design features have already been specified in the XML files. The user is then asked to specify the relevant parameters, as was described above, and the Wizard then generates the appropriate the VBScript file and creates a launching icon that appears on the Windows desktop. This feature enables the experimenter to launch the program with a single mouse-click.

A second feature of the Wizard enables the user to modify the categorical data that are selected by predefined parameters. The parameters and their associated categories are stored in a special XML file (ANOTMaster.xml). Each category has both a text and a numerical representation. The categorical data, created by the coders, are stored in the coders' result XML file. The Wizard allows these data to be converted into a text-delimited format for importation into statistical packages, such as SPSS.

\section{COMPARISON WITH OTHER SOFTWARE}

For decades, computers have been used to record and process the data derived from observations of human interactions and behavior (Crossman, Williams, \& Chambers, 1978; Deni, Szijarto, Eisler, \& Fantauzzo, 1983; Flowers, 1982; Markman \& Poltrock, 1982). Most older data-recording systems, such as the Observer (Noldus, 1991), ODAP (Hetrick, Isenhart, Taylor, \& Sandman, 1991), and MOOSES (Tapp, Wehby, \& Ellis, 1995), were designed for use in direct observation studies. Although these systems can be used in studies in which data coders watch and record behaviors captured on videotape, they fail to provide an adequate synchronization capability.

In contrast, more recent systems offer the possibility of interfacing a VCR with a computer using computerreadable time code on the tape (Noldus, Trienes, Hendriksen, Jansen, \& Jansen, 2000). They also enable control of the start/stop position of the videotape by the computer. Systems that offer these possibilities include the PROCODER (Tapp \& Walden, 1993) and the Observer Video-Pro (Noldus et al., 2000). Other tools that support the annotation and retrieval of video data are the VIDEOLOGGER (Krauss, Morrel-Samuels, \& Hochberg, 1988), VideoNoter (Roschelle \& Goldman, 1991), VTLOGANL (Hecht \& Roberts, 1996), and ObsWin32 (Martin, Oliver, \& Hall, 1999), an observationaldata acquisition program that works with digital video (MPEG or AVI).

The main disadvantage of this type of computercontrolled VCR approach is the need for expensive VCRs. Only the most recent systems, such as the Observer VideoPro and ObsWin32, make it possible to use modern multimedia techniques. Camera images can be recorded directly into files on the PC in compressed format (MJPEG or MPEG-1). Replay precision of up to one or a few frames can be controlled directly by the software. MJPEG and MPEG compression can currently be achieved with inexpensive hardware, and modern PCs offer sufficient storage capacity for this purpose (10-40 Gbyte).

Although some of the systems mentioned, such as the Observer Video-Pro, offer excellent performance in behavioral observation studies, they are designed to systematize the often enormous task of data coding and data analysis (Hecht \& Roberts, 1996) in studies for which highly trained raters are available. The raters' behavioral observations are typically coded by entering special key sequences or by using a mouse, a process that can require extensive training and special skills. In cases like this, special instructional software, such as COR (Blasko, Kaz- 
merski, Corty, \& Kallgren, 1998), can be used to train the raters to make and record the appropriate observations. Special training sessions for using the Observer system are also available.

On the other hand, the skills required to use this type of system cannot be expected from research participants who have minimal computer knowledge or who, in the case of certain subpopulations, such as people with autism, are cognitively compromised. VIDANN, the system described here, has been explicitly designed for such inexperienced users. In our view, VIDANN should not be viewed as a competitor of the Observer, Procoder, and other existing video analysis packages. VIDANN is not, in itself, a fully integrated video-based observational data acquisition software package. Instead, VIDANN is a sophisticated computer-controlled notepad that permits the integration of videotaped interaction sequences with the corresponding comments made by the participants when they watch themselves on the videotape.

A similar result could be obtained using the Observer system as well, but with the important limitation that extensive training would be required. Unlike the Observer system, VIDANN is not intended to be used by trained observers in order to obtain quantitative records of behavior. Instead, VIDANN preserves verbal behavior (in the form of writing) and links it to parts of a video. In contrast, observational data acquisition software sytems are typically used to facilitate the coding (i.e., measuring) of observed behaviors, whether they are verbal (conversation, writing) or nonverbal (facial expressions, hand gestures, etc.).

\section{DISCUSSION}

The VIDANN system, as was described above, offers a number of advantages, as compared with traditional VCR recording. First, there is no inaccuracy related to the use of a manual remote control system to stop a videotape. This inaccuracy can be resolved by using a professional VCR with a built-in RS232 interface (Noldus et al., 2000), but recording directly to the computer disk can be achieved at about one tenth of the price. Second, the use of a writing tablet eliminates the time-consuming activity of scanning or transcribing a large number of written records. Third, participants (including those with impaired cognitive functioning, such as people with autism) are not required to have any direct contact with the computer. They simply watch a videotape and write down their reactions on paper, as they are used to doing in their note-taking activities in everyday life. Fourth, the experimenter can watch the participant's writing as it appears onscreen and restart the videotape if necessary. In contrast, existing computer systems, such as the Observer Video-Pro (Noldus et al., 2000), are designed for professionals and are too sophisticated for untrained participants to use.

The VIDANN program was created as a set of modular objects that can be used in any program in which COM objects can be incorporated. The COM objects in VIDANN employ a Visual Basic script (VBScript) that is executed by the Microsoft scripting host, but it should be possible to write them in other scripting languages, such as Java script, as well. VIDANN has been made userfriendly to the experiment designer by adding a Wizard that generates the appropriate VBScript. This script contains all of the necessary parameter definitions, a feature that helps to ensure that any design and programming errors will be minimized.

Although the VIDANN system was designed for empathic accuracy research, it can readily be adapted to any psychological experiments in which participants are required to observe video segments with predefined stops and make written annotations on paper. It is possible, for example, to predefine a series of either random stops or fixed-interval stops by entering the exact stop times in seconds, instead of asking the participants to use the start/ stop button to designate points at which specific cognitive or behavioral events occurred. It would also be possible to adapt the software to allow for audio input. Another option may be to link the data saved by VIDANN with data provided by the Observer or other video analysis systems. Such an option would make it possible to relate the quantitative analysis of the kind of sequences of behavior that are gathered by, for example, the Observer to a text analysis of the written notes taken with VIDANN.

All of the above-described software is free and can be downloaded from http://twiprof1.rug.ac.be/VIDANN. The VIDANN source code can be obtained from armand. declercq@rug.ac.be.

\section{REFERENCES}

Blasko, D. G., Kazmerski, V. A., Corty, E. W., \& Kallgren, C. A (1998). Courseware for observational research (COR): A new approach to teaching naturalistic observation. Behavior Research Methods, Instruments, \& Computers, 30, 217-222.

Cover, R. (1999). Introducing the Extensible Markup Language (XML) [On-line]. Available: http://www.oasis-open.org/cover/xmlIntro.html. Crossman, E. K., Williams, J. G., \& Chambers, J. H. (1978). Using the PET microcomputer for collecting and analyzing observational data in the classroom. Behavior Research Methods \& Instrumentation, 10, 563-566.

De ClercQ, A., Desoete, A., \& Roeyers, H. (2000). EPA2000: A multilingual, programmable computer assessment of off-line metacognition in children with mathematical-learning disabilities. Behavior Research Methods, Instruments, \& Computers, 32, 304-311.

Deni, R, Szijarto, K., Eisler, A., \& Fantauzzo, C. (1983). BASiC programs for observational research using the TRS-80 Model 100 portable and Model 4 computers. Behavior Research Methods \& Instrumentation, 15, 616.

Flowers, J. H. (1982). Some simple Apple II software for the collection and analysis of observational data. Behavior Research Methods \& Instrumentation, 14, 241-249.

Hecht, J. B., Roberts, N. K. (1996). VTLOGANL: Coding and analyzing videotaped data. Behavior Research Methods, Instruments, \& Computers, 28, 76-82.

Hetrick, W. P., Isenhart, R. C., Tay lor, D. V., \& Sandman, C. A. (1991). ODAP: A stand-alone program for observational data acquisition. $\mathrm{Be}$ havior Research Methods, Instruments, \& Computers, 23, 66-71.

Ickes, W., Stinson, L., Bissonnette, V. L., \& Garcia, S. (1990). Naturalistic social cognition: Empathic accuracy in mixed-sex dyads. Journal of Personality \& Social Psychology, 59, 730-742.

Krauss, R. M., Morrel-Samuels,P., \& HochberG, J. (1988). VIDEOLOGGER: A computerized multichannel event recorder for analyzing videotapes. Behavior Research Methods, Instruments, \& Computers, 20, 37-40. 
Markman, H. J., \& Poltrock, S. E. (1982). A computerized system for recording and analysis of self-observations of couples' interaction. $B e$ havior Research Methods \& Instrumentation, 14, 186-190.

Martin, N., Oliver, C., \& Hall, S. (1999). ObsWin: Observational data collection and analysis for Windows. CTI Psychology Software News, 9, 14-16.

Noldus, L. P. J. J. (1991). The Observer: A software system for collection and analysis of observational data. Behavior Research Methods, Instruments, \& Computers, 23, 415-429.

Noldus, L. P. J. J., Trienes, R. J. H., Hendriksen, A. H. M., Jansen, H., \& JANSEN, R. G. (2000). The Observer Video-Pro: New software for the collection, management, and presentation of time-structured data from videotapes and digital media files. Behavior Research Methods, Instruments, \& Computers, 32, 197-206.

Okstein, C. J. (1999). XML: A key technology for sharing clinical information. M D Computing, 16, 31-33.

Roschelle, J., \& Goldman, S. (1991). VideoNoter: A productivity tool for video data analysis. Behavior Research Methods, Instruments, \& Computers, 23, 219-224.

SCHNEIDER, W. (1998, November). E-Prime: A cross platform experiment generator studio for computerized behavioral research. Paper presented at the Commercial Symposium of the 28th Annual Conference of the Society for Computers in Psychology, Dallas.

Spencer, P. (1999). Professional XML design and implementation. Birmingham, U.K.: Wrox Press.

StockBUrger, D. W. (1999, November). Dynamic data analysis in online publications using extensible markup language (XML) data islands and dynamic hypertext markup language (DHTML). Paper presented at the 29th Annual Meeting of the Society for Computers in Psychology, Los Angeles.

TAPP, J., \& WALDEN, T. (1993). PROCODER: A professional tape control, coding, and analysis system for behavioral research using videotape. Behavior Research Methods, Instruments, \& Computers, 25, 53-56.

TAPP, J., WehBy, J., \& Ellis, D. (1995). A multiple option observation system for experimental studies: MOOSES. Behavior Research Methods, Instruments, \& Computers, 27, 25-31.

\section{NOTE}

1. In the present version of VIDANN, participants do not have the option to replay any part of the tape in this phase. If necessary, this option can be included in a subsequent version of the system.

\section{APPENDIX \\ Listing of Coder.VBS}

A brief explanation can be viewed at http://twiprof1.rug.ac.be/VIDANN/VBScript.htm.

Const CodeFile $=\& \mathrm{H} 10$

Const CodeOverWrite $=5$

Const AllWithoutExternalTimingFromThis Video $=4$

Const AllWithExternalTimingFromThisXMLFile $=5$

Const ExternalTiming $=3$

Dim GO, f, V, I, F1, F2, vstop, videofile

Set GO = CreateObject("VIDANN.GO")

Set V = CreateObject("VIDANN.MARK")

GO.SysPath " "

GO.MessageFile "MessageEN.XML"

GO.Parameters "WindowPar.XML"

GO.SetVideoKeys "F12", "F1", " "," "

GO.AVIType "AVIVIDEO"

GO.SetWindowLabel "Start the video by pressing F12"

GO.FramesPerSecond 25

GO.DefaultDataFileDirectory "D:\Gbr_BCK_WRKIWRK।" 16

GO.UseWindow "FAVI", "FAVIEX"

GO.UseWindow "FTAB1", "FTAB1EX"

GO.UseWindow "FTAB2", "FTAB2EX" 18

GO.UseWindow "FCODE", "FCODE" 20

videofile = GO.GetVideoFile(" ") 21

GO.OpenOutputFile CodeFile, CodeOverWrite, VideoFile, " " 22

F1 = GO.SelectInputFile(AllWithoutExternalTimingFromThisVideo, VideoFile) 23

F2 = GO.SelectInputFile(AllWithExternalTimingFromThisXMLFile, F1) 24

GO.OpenInputFile "FTAB1", F1, 1

GO.OpenInputFile "FTAB2", F2, 2

GO.SetTimingData F1, V 27

for $\mathrm{i}=1$ to 256

VStop $=$ GO.DisplayVideo(ExternalTiming, V.VStop(i)) 29

If vstop $<0$ Then Exit for ' stop $\quad 30$

GO.DisplayWindow 1, vstop $\quad 31$

GO.DisplayWindow 2, vstop $\quad 32$

GO.AcceptInput vstop, $0 \quad 33$

NEXT

GO.CloseAll $\quad 35$

set $\mathrm{V}=$ nothing $\quad 36$

set $\mathrm{GO}=$ nothing $\quad 37$

(Manuscript received November 8, 2000;

accepted for publication January 28,2001 .) 